\title{
A Preliminary Study on the Application of FEM in Financial Engineering Analysis
}

\author{
Yang Yan- $\mathrm{xi}^{1}{ }^{\text {, a }}$ \\ ${ }^{1}$ Party School of the Organ Directly Under the Hunan CPC Provincial Committee, \\ Changsha, Hunan, 410079, China \\ agvletter@sina.com
}

Keywords: Financial Engineering; Numerical Analysis; Finite Element Method; Finite Difference Method; Stochastic Differential Equations

\begin{abstract}
Many practical problems in modern finance can be cast into the framework of stochastic differential equations, so that numerical techniques from engineering can be adapted. The static 1D problem in financial engineering characterized by non-self-adjoint is examined in this paper using the Finite Element Method (FEM). The finite element for the problem mentioned above is established based on Galerkin criterion. And numerical examples, such as first exit time of a geometric Brownian motion, and convection-dominated problems, are solved using FEM. The results are compared with analytic solution, FDM respectively. It is shown that high computational accuracy and efficiency are achieved using FEM and this method can be further used in dynamic problem, 2D problem of financial engineering.
\end{abstract}

\section{Introduction}

In modern finance, many practical problems can be cast into the framework of stochastic differential equations, so that numerical techniques from engineering can be adapted [1,2]. Differential equations have been studied for some centuries by mathematicians, physicists and engineers, so that a great deal of knowledge from these areas is available. Techniques for finding numerical solutions for differential equations arising in finance are the topic of this paper. The Method of Finite Elements (FEM) has been put forward through a number of academic papers focusing on structures analyses[3-8]. Here, we want to use the Galerkin FEM, one of the Weighted Residual Methods, to get the numerical solutions of 1D static problem in finance engineering which is characterized by non-self-adjoint.

\section{Galerkin Finite Element}

A general two-point boundary value problem arising in economics which, furthermore, is not self-adjoint[3]:

$$
\begin{gathered}
u^{\prime \prime}(x)+a_{1}(x) u^{\prime}(x)+a_{2}(x) u(x)+f(x)=0 \\
\alpha_{1} u\left(x^{\min }\right)+\beta_{1} u^{\prime}\left(x^{\min }\right)=\gamma_{1} \\
\alpha_{2} u\left(x^{\max }\right)+\beta_{2} u^{\prime}\left(x^{\max }\right)=\gamma_{2}
\end{gathered}
$$

$u(x)$ is the unknown function defined during $\left(x^{\min }, x^{\max }\right)$ in equation $(1), u^{\prime \prime}(x) 、 u^{\prime}(x)$ are the 1 st order and 2nd order derivartives respectively. Equation (2), (3) are the boundary conditions.

The Galerkin criterion requires that the following expression vanishes:

$$
\int_{x^{\min }}^{x^{\max }} \delta u\left[u^{\prime \prime}+a_{1} u^{\prime}+a_{2} u+f\right] d x=0
$$


$\delta u$ is the variation of $u$.To eliminate the second order derivative $u^{\prime \prime}(x)$, the first term from the integral in equation (4) is integrated by parts:

$$
\begin{aligned}
& \int_{x^{\min }}^{x_{\max }} \delta u\left[u^{\prime \prime}+a_{1} u^{\prime}+a_{2} u+f\right] d x=\left.u^{\prime} \delta u\right|_{x^{\min }} ^{x_{\max }}-\int_{x^{\min }}^{x^{\max }} u^{\prime} \delta u^{\prime} d x+\int_{x^{\min }}^{x^{\max }} \delta u\left[a_{1} u^{\prime}+a_{2} u+f\right] d x \\
= & \int_{x^{\min }}^{x^{\max }}\left[-u^{\prime} \delta u^{\prime}+a_{1} u^{\prime} \delta u+a_{2} u \delta u+f \delta u\right] d x+b \cdot t \cdot(u)=0
\end{aligned}
$$

b.t. $(u)$ is the boundary conditions.

Assuming that an approximate solution between two nodes $x_{\mathrm{i}}, x_{\mathrm{i}+1}$ can be represented by a straight line, linear interpolation is employed on this interval:,

$$
u^{e}(x)=a x+b
$$

Both $a$ and $b$ are to be chosen in a way that $u^{e}\left(x_{i}\right)=u_{i}$ and $u^{e}\left(x_{i+1}\right)=u_{i+1}$. This results in:

$$
u=\mathbf{N u}^{(e)} \text {. }
$$

$\mathbf{N}$ is called elemental linear interpolation functions or shape funtions.

Substituting the approximate function Equation (7) into the boundary value problem given by Equation (5) leads to:

$$
\begin{aligned}
& \int_{x_{i}}^{x_{i+1}}\left[-u^{\prime} \delta u^{\prime}+a_{1} u^{\prime} \delta u+a_{2} u \delta u+f \delta u\right] d x \\
& =\delta \mathbf{u}^{(e) T}\left(\int_{x_{i}}^{x_{i+1}}\left(-\mathbf{N}^{\prime T} \mathbf{N}^{\prime}+a_{1} \mathbf{N}^{T} \mathbf{N}^{\prime}+a_{2} \mathbf{N}^{T} \mathbf{N}\right) d x\right) \mathbf{u}^{(e)} \\
& \quad+\delta \mathbf{u}^{(e) T}\left(\int_{x_{i}}^{x_{i+1}} \mathbf{N}^{T} f d x\right) \\
& =-\delta \mathbf{u}^{(e) T} \mathbf{K}^{(e)} \mathbf{u}^{(e)}+\delta \mathbf{u}^{(e) T} \mathbf{F}^{(e)} .
\end{aligned}
$$

where

$$
\begin{aligned}
& \mathbf{K}^{(e)}=\int_{x_{i}}^{x_{i+1}}\left(-\mathbf{N}^{\prime T} \mathbf{N}^{\prime}+a_{1} \mathbf{N}^{T} \mathbf{N}^{\prime}+a_{2} \mathbf{N}^{T} \mathbf{N}\right) d x \\
& \mathbf{F}^{(e)}=\int_{x_{i}}^{x_{i+1}} \mathbf{N}^{T} f d x
\end{aligned}
$$

Finally, the equation (5) can be written as

$$
\begin{aligned}
& \int_{x^{\min }}^{x_{\max }} \delta u\left[u^{\prime \prime}+a_{1} u^{\prime}+a_{2} u+f\right] d x \\
= & -\delta \mathbf{u}^{(e) T} \mathbf{K}^{(e)} \mathbf{u}^{(e)}+\delta \mathbf{u}^{(e) T} \mathbf{F}^{(e)}+\text { b.t. }(u)=0
\end{aligned}
$$

So the equation (10) can be simplified due to the arbitrariness of $\delta \mathbf{u}^{(e)}$,

$$
\mathbf{K}^{(e)} \mathbf{u}^{(e)}=\mathbf{F}^{(e)}
$$

each elemental matrix $\mathbf{K}^{(e)}$ and vector $\mathbf{u}^{(e)}, \mathbf{F}^{(e)}$ can be expanded to the global ones as follows as the same as classical FEM:

$$
\mathbf{K u}=\mathbf{F}
$$

As to the boundary contions b.t. $(u)$, when $\beta_{1} \neq 0$ and $\beta_{2} \neq 0$, b.t. $(u)$ can be written as

$$
\text { b.t. }(u)=\left.u^{\prime} \delta u\right|_{x^{\min }} ^{x_{\max }}=\delta u_{N} \frac{\gamma_{2}-\alpha_{2} u_{N}}{\beta_{2}}-\delta u_{1} \frac{\gamma_{1}-\alpha_{1} u_{1}}{\beta_{1}}
$$

The global matrix $\mathbf{K}$ 、 global vector $\mathbf{F}$ could be updated as: 


$$
\begin{aligned}
& \hat{\mathbf{K}}_{11}=\mathbf{K}_{11}+\frac{-\alpha_{1}}{\beta_{1}}, \quad \hat{\mathbf{K}}_{N N}=\mathbf{K}_{N N}+\frac{\alpha_{2}}{\beta_{2}} \\
& \hat{\mathbf{F}}_{1}=\mathbf{F}_{1}+\frac{-\gamma_{1}}{\beta_{1}}, \quad \hat{\mathbf{F}}_{N}=\mathbf{F}_{N}+\frac{\gamma_{2}}{\beta_{2}}
\end{aligned}
$$

The rest elements of $\hat{\mathbf{K}}, \hat{\mathbf{F}}$ are consistent with $\mathbf{K}, \mathbf{F}$, so the equation (12) can be written as

$$
\hat{\mathbf{K}} \mathbf{u}=\hat{\mathbf{F}} \text {. }
$$

When $\beta_{1}=0$ and $\beta_{2}=0$, b.t. $(u)$ can be written as

$$
\text { b.t. }(u)=\left.u^{\prime} \delta u\right|_{x^{\min }} ^{x_{\max }}=\delta u_{N} u_{N}^{\prime}-\delta u_{1} u_{1}^{\prime}
$$

Because of $\beta_{1}=0$ and $\beta_{2}=0, u_{1} 、 u_{N}$ are constants,

$$
u_{1}=\frac{\gamma_{1}}{\alpha_{1}}, \quad u_{N}=\frac{\gamma_{2}}{\alpha_{2}}
$$

so

$$
\delta u_{1}=\delta u_{N}=0
$$

The global matrix $\mathbf{K}$ 、 global vector $\mathbf{F}$ could be updated as:

$$
\begin{aligned}
& \hat{\mathbf{K}}_{11}=1, \quad \hat{\mathbf{K}}_{1 j}=0, j=2, \cdots, N . \\
& \hat{\mathbf{K}}_{N N}=1, \hat{\mathbf{K}}_{N j}=0, j=1, \cdots, N-1 . \\
& \hat{\mathbf{F}}_{1}=\frac{\gamma_{1}}{\alpha_{1}}, \quad \hat{\mathbf{F}}_{N}=\frac{\gamma_{2}}{\alpha_{2}}
\end{aligned}
$$

The rest elements of $\hat{\mathbf{K}}, \hat{\mathbf{F}}$ are are consistent with $\mathbf{K}, \mathbf{F}$, so the equation (12) can be also written as

$$
\hat{\mathbf{K}} \mathbf{u}=\hat{\mathbf{F}}
$$

The other boundary condition, like $\beta_{1} \neq 0$ and $\beta_{2}=0$, can be treated similarly.

The unknown variables $\mathbf{u}$ can be obtained after solving the linear algebraic equations (15), (20).

\section{Numerical Results}

To illustrate the effectiveness of the FEM in analysis of 1D static problem in finance engineering, numerical examples are studied in this section.

First exit time of a geometric Brownian motion. We first compute numerically the expected exit time of a geometric Brownian motion[9]

$$
d Y=a Y d t+\sigma Y d X
$$

with $\mathrm{a}=0.1$ and $\sigma=0.2$ leaving the region $[20,60]$. In practice, such a problem arises when one wants to know how long it takes for a given asset to leave a certain corridor for the first time. This information can be useful, for instance, in assessing the risk of a double barrier option.

Labeling the independent variable as $x$, this problem can be formulated as an ODE

$$
a x u^{\prime}+\frac{\sigma^{2}}{2} x^{2} u^{\prime \prime}=-1 \text { with } u\left(x^{\min }\right)=0, u\left(x^{\max }\right)=0 .
$$

The analytical solution to the problem (22) is given by[3]:

$$
u(x)=\frac{1}{\sigma^{2} / 2-a}\left(\ln \left(\frac{x}{x^{\min }}\right)-\frac{1-\left(x / x^{\min }\right)^{1-2 a / \sigma^{2}}}{1-\left(x^{\max } / x^{\min }\right)^{1-2 a / \sigma^{2}}} \ln \left(\frac{x^{\min }}{x^{\max }}\right)\right) .
$$


Table 1 First exit time of a geometric Brownian motion

\begin{tabular}{ccc}
\hline Asset price $x$ & Analytical solution & Numerical solution \\
\hline 20.0 & 0.00000000 & 0.00000000 \\
25.0 & 5.41981128 & 5.41981128 \\
30.0 & 6.08946721 & 6.08946721 \\
35.0 & 5.42660555 & 5.42660555 \\
40.0 & 4.37095254 & 4.37095254 \\
45.0 & 3.22515949 & 3.22515949 \\
50.0 & 2.09472725 & 2.09472725 \\
55.0 & 1.01618148 & 1.01618148 \\
60,0 & 0.00000000 & 0.00000000 \\
\hline
\end{tabular}

The finite element discretization consists of 200 equal-size elements with linear basis function.The numerical results compared with analytical solution are listed in Table 1. It is seen that the finite element formulations are able to provide a very good estimates of the first exit time.

Convection-dominated problems. Recently, various publications dealing with convection-dominated problems in finance have appeared [10]. In equity and FX option pricing convection-dominated problems arise because of low asset prices and/or low volatilities. We present the numerical problems arising in convection-dominated problems with the help of an example. Consider the following boundary value problem

$$
-k u^{\prime \prime}+u^{\prime}=0 \text { with } u(0)=0, u(1)=1 .
$$

For small $k$ the above differential equation loses its elliptic character and starts to resemble a hyperbolic problem. Then, Equation (24) is called convection-dominated because the convection term $u$ ' dominates the character of the problem. Although Equation (24) is still elliptic by definition, techniques for elliptic problems tend to fail for decreasing $k$. This problem is solved by:

$$
u(x)=\left(1-e^{(x / k)}\right) /\left(1-e^{(1 / k)}\right) .
$$

The finite element discretization consists of 200 equal-size elements with linear basis function.The numerical results when $k=0.002$ compared with analytical solution and finite difference method (FDM) are depicted as Fig.1. It is seen that the FDM solution exhibits an oscillation (blue box) while the FEM solution provide acceptable predictions for convection-dominated problems.

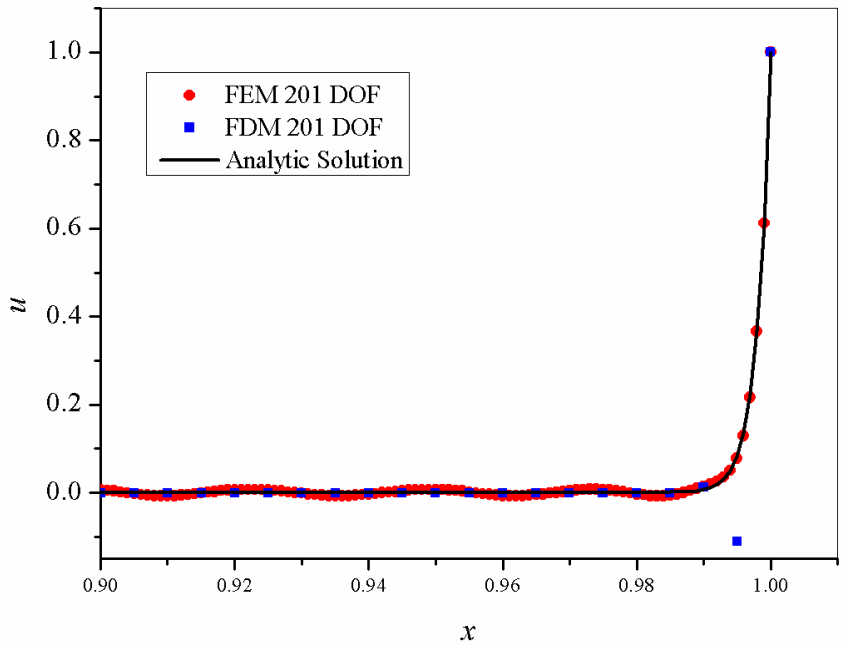

Figure. 1 Results of a convection-dominated example with $k=0.002$ 


\section{Conclusion}

The static 1D problem in financial engineering characterized by non-self-adjoint is examined in this paper using the Finite Element Method. The finite element for the problem mentioned above is established and numerical results from FEM are compared with analytic solution, FDM respectively. It is shown that high computational accuracy and efficiency are achieved using FEM and this method can be further used in dynamic problem, 2D problem of financial engineering.

\section{References}

[1] M. S. Ross: An Elementary Introduction to Mathematical Finance (Cambridge University Press, London 2011).

[2] P. Wilmott, J.Dewynne and S. Howison: Option Pricing: Mathematical Models And Computation (Oxford Financial Press, Oxford 1994).

[3] J. Topper: Financial engineering with finite elements (John Wiley \& Sons Ltd, Chichester 2005).

[4] M. Sajid: Journal of Numerical Analysis and Modeling, Series B, Vol. 3 (2012), No.3, p345.

[5] X. Wu, Z. Ding. Numerical Methods for Partial Differential Equations, Vol. 18 (2002), No. 6, p711.

[6] M. Fatica, E. Phillips. Proceedings of the 6th Workshop on High Performance Computational Finance (Turin, Italy, November, 2013) p5.

[7] W Li, S Wang. Journal of Industrial and Management Optimization, Vol. 9 (2013), No.2. p365.

[8] S Salmi, J Toivanen. International Journal of Computer Mathematics, Vol. 89(2012), No.9. p1112.

[9] A. C. Chiang. Elements of Dynamic Optimization (McGraw-Hill, Inc, New York 1992).

[10]R.Seydel. Tools for Computational Finance (Springer, Berlin 2002). 